\title{
Lexical Networks and Lexicon Profiles in Didactical Texts for Science Education
}

\section{Koponen, Ismo}

Springer International Publishing AG

2020

Koponen , I \& Nousiainen, M 2020 , Lexical Networks and Lexicon Profiles in Didactical Texts for Science Education . in H Cherifi , S GaitoJosé , J F Mendes , E Moro \& L M Rocha (eds), Complex Networks and Their Applications VIII : Volume 2 Proceedings of the Eighth International Conference on Complex Networks and Their Applications COMPLEX NETWORKS 2019 . vol. 1, Studies in Computational Intelligence, vol. 882 , Springer International Publishing AG , Cham , pp. 15-27 , Complex Networks , Lisbon , Portugal , 10/12/2019 . https://doi.org/10.1007/978-3-030-36683-4_2

http://hdl.handle.net/10138/314437

https://doi.org/10.1007/978-3-030-36683-4_2

unspecified

acceptedVersion

Downloaded from Helda, University of Helsinki institutional repository.

This is an electronic reprint of the original article.

This reprint may differ from the original in pagination and typographic detail.

Please cite the original version. 


\title{
Lexical Networks and Lexicon Profiles in Didactical Texts for Science Education
}

\author{
Ismo T. Koponen $\left.{ }^{(}\right)$and Maija Nousiainen \\ Department of Physics, University of Helsinki, P.O. Box 64, 00014 Helsinki, Finland \\ ismo.koponen@helsinki.fi
}

\begin{abstract}
The lexical structure of language of science as it appears in teaching and teaching materials plays a crucial role in learning the language of science. We inspect here the lexical structure of two texts, written for didactic purposes and discussing the topic of wave-particle dualism as it is addressed in science education. The texts are analyzed as lexical networks of terms. The analysis is based on construction of stratified lexical networks, which allows us to analyze the lexical connections from the level of cotext (sentences) to context. Based on lexical networks, we construct lexicon profiles as they appear in two texts addressing the wave-particle dualism of electrons and photons. We demonstrate that the lexicon profiles of the two texts, although they discuss the same topic with similar didactic goals, nevertheless exhibit remarkable variation and differences. The consequences of such variation of lexicon profiles for practical teaching are discussed.
\end{abstract}

Keywords: Lexical network $\cdot$ Lexicon learning $\cdot$ Science education

\section{Introduction}

The structure of the language of science as it appears in science teaching and instruction is a widely researched topic owing to its importance in introducing students to the proper ways in which to use the vocabulary and syntax of scientific language [1-4]. The structure of scientific language in learning has often been approached by examining the structure of networks formed by terms that stand for concepts. Most often, this approach is referred as analysis of semantic networks, where meaning of words and terms is assumed to emerge from their mutual connections [2,5-8]. Such an approach to study role of scientific language in learning founds support from two directions: from analysis of scientific knowledge and from cognitive linguistics.

Analysis of the structure of scientific knowledge suggests, according to Kuhn [9], that it is built essentially in the form of lexical networks, as a lexicon of terms, where connections between the terms derive from contextualized instances of how terms are used. Such a notion of lexical networks and lexicons was central to the conception of scientific knowledge that Kuhn developed later in his research [10]. 
For Kuhn, lexicons also define scientific communities, because the individual members of the communities must share substantial parts of the lexicons to be able to communicate with and provide identity to the community $[9,10]$.

Focus on lexical networks also finds support from the research on how the meaning of words is learned $[11,12]$. It has been pointed out that learning the meaning of words involves lexical networks made of names and words and the semantic connections between them, which build upon the conceptual system but are different from it. Thus, the conceptual system is not directly accessible in communication, in the form of written or spoken knowledge, but lexical networks and semantic connections provide access points to it [11]. In this view, the three levels - lexical, semantic and conceptual - are understood as distinct but related levels.

Some researchers maintain that representations encoded by language can be equated with semantic meanings [12], while some others see these linguistic and semantic structures as different [11]. From the viewpoint of lexicons and the role of lexical networks as adopted here, several studies, which have framed their targets as students' semantic networks, have in fact focused on the lexical networks rather than on semantic networks $[2,5-8,13,14]$. The difference between the lexical and semantic networks, however, is not crucial here but for reasons of clarity, we have chosen to retain the distinction and frame our target as the analysis of lexical rather than semantic networks.

The lexical network approach to analyzing written knowledge provides practical tools to develop effective operationalizations to study the structure of lexical networks and lexicons in learning. We focus here on didactical texts meant for science education, and investigate how different lexicons appear in them. The decision to pay attention on didactical texts derives from the notion that the vocabulary of didactical texts like textbooks may have a crucial role in learning (see e.g. [2] and references therein). As a target of the study, we have chosen two well-known texts about a topic that has recently raised interest in science education: the wave-particle dualism of electrons and photons as quantum entities, which is known to raise much confusion not only in learning the topic but also how different interpretations of dualism infiltrate the process of teaching it $[15,16]$. The texts we have chosen to study are: (A) a text that introduces electrons and photons as field quanta, discusses them both quite similarly as field excitations and approaches wave-particle dualism from this viewpoint [17]; (B) another text, which takes a viewpoint emphasizing the role of statistical interpretation of measurements and statistical (or ensemble) understanding of the quantum nature of measurement events [18]. The differences between these views and their consequences for didactics are well recognized [15]. Therefore they are good candidates to test the method of analyzing the lexical structure in didactical texts which have similar similar goal, scope and intended audience, but supposedly differences in their vocabulary. 


\section{Method}

The two didactical texts $\mathrm{A}$ and $\mathrm{B}$ studied here are analysed stepwise: first, the texts are pruned to create the text-corpus to be analyzed; second; a stratified lexical network is constructed to reveal the connectivity of words and terms in different levels; third and finally, a lexicon profile that condenses the important terms is formed using a network-based analysis.

\subsection{Construction of Text Corpus}

The parts of texts in $\mathrm{A}$ and $\mathrm{B}$ to be analyzed have a common theme of waveparticle dualism (WPD). The text excerpts consist of about 1200 words in A and 1000 in B. In both texts, the double-slit experiment for weak intensity light and electron beams play a crucial role, thus setting the broad context in which to discuss WPD. This body of text is divided into 11 narrower, roughly similar, contexts (denoted by $\mathrm{KK}$ in what follows), including the preliminary introduction of the topic $\left(\mathrm{KK}_{1}\right)$, discussion of experimental apparatus $\left(\mathrm{KK}_{2}\right)$, discussions of details of the experiments and observations $\left(\mathrm{KK}_{3}-\mathrm{KK}_{5}\right)$, qualitative interpretations $\left(\mathrm{KK}_{6}-\mathrm{KK}_{8}\right)$, theoretical explanations $\left(\mathrm{KK}_{9}-\mathrm{KK}_{10}\right)$ and summary of main conclusion $\left(\mathrm{KK}_{11}\right)$. Each context $\mathrm{KK}$ contains from one to four cotexts $(\mathrm{K})$, in which sentences form units discussing some sub-topic within the context KK. Within the cotexts $\mathrm{K}$, the text is pruned by reducing the sentences to main clauses $v$. The nouns $n$ appearing in the clauses are then found and listed. For clearly synonymous nouns, a common noun is used. In addition, the clauses are roughly classified according to their modality: Questions (Q), assertions (A) and conclusions $(\mathrm{C})$.

The lexicons of interest here are related to terms $\mathrm{T}=[$ electron] and $\mathrm{T}=$ [photon], where brackets indicate that we treat them as lexical expressions. The lexicon is formed by all those terms and words that are linked to $T$ through the lexical network. Instead of a complete lexicon, the goal is to form a condensed representation of it, in the form of a lexicon profile. The lexicon profiles are formed in four steps by:

1. Constructing the stratified lexical network from clauses $v$ to contexts $K K$.

2. Finding the most relevant terms through counting weighted walks.

3. Constructing lexical proximity network on the basis of walks.

4. Extracting the key terms which form the lexicon profile for $\mathrm{T}$.

\subsection{Construction of Lexical Networks}

The construction of stratified lexical networks is performed so that we can differentiate levels from single clauses to the cotext of several clauses and finally up to context. To accomplish this, the pruned text consisting of main clauses is transformed into a network in which nodes corresponding to relevant terms and words (nouns) $n$ are first connected to nodes representing a root verb $v$. The root verb nodes $v$ are next connected to nodes $V_{\mathrm{X}}$ that represent the modality 
of clause $\mathrm{X} \in\{\mathrm{Q}, \mathrm{A}, \mathrm{C}\}$, and are then connected to cotext $\mathrm{K}$. These connections are clarified in scheme in Table 1 . Note that $V_{\mathrm{Q}}$ and $V_{\mathrm{C}}$ are connected to $V_{\mathrm{A}}$ as shown in Table 1 since modalities $\mathrm{Q}$ and $\mathrm{C}$ also require assertion-type clauses A to be meaningful. After making these connections, the cotexts are connected to contexts KK and finally, connections between contexts KK are made to form the complete structure of the text. The part of the network which consists of connections from set of nouns $\{n\}$ up to cotext $\mathrm{K}$ and context $\mathrm{KK}$ is shown in Table 1 with length of walks $\mathrm{L}$ needed to reach the term $\mathrm{T}$ from given noun $n$. Note that $\mathrm{T}$ is part of set $\{n\}$ but reversed link $v \rightarrow T$.

Table 1. The construction of lexical networks. The form of links are shown in column "Link". The length $\mathrm{L}$ of walk that connects a given noun $n$ to term $\mathrm{T}$ of interest is given in column "Walk". Note that the term $\mathrm{T}$ is not shown, but it would be a link $v \rightarrow T$.

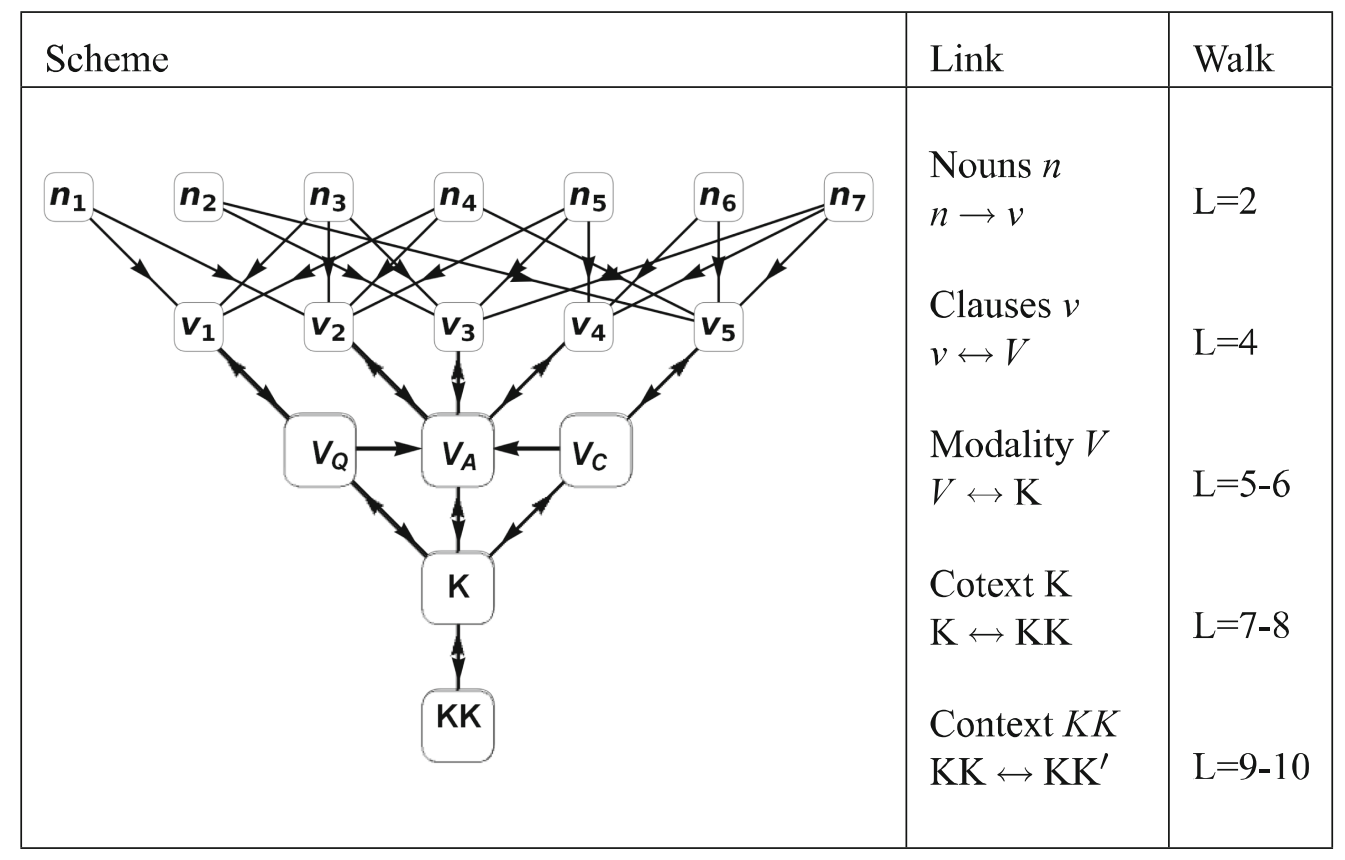

\subsection{Analysis of the Network}

The better the global connectivity between terms and words (nouns) in the network based on counting the walks between them, the more relevant we consider the connections between them. This decision to attach relevance to terms is different from the standard closeness criteria used to construct lexical (or semantic) networks $[2,5,6]$. However, a measure which takes into account the global connectivity of nodes in the stratified network has more resolving power with regard to the level of connections. We quantify such connectivity here by using communicability [19-21]. Closeness, however, will be relevant later on when we have constructed the lexical proximity network.

The lexical network can be described by a $N x N$ adjacency matrix $\mathbf{A}$ with elements $[A]_{p q}=a_{p q}$, where $a_{p q}=1$ when nodes are connected and $a_{p q}=0$ 
when they are not connected. The powers $k$ of adjacency matrix $\mathbf{A}$ can be used to obtain the number of walks of length $k$ connecting two nodes within the network. In a connected network, however, the number of long walks increases rapidly, nearly factorially with the length of the walk. Therefore, the number of walks is usually divided by the factorial, to obtain the communicability [19-21]. For the walk counting, we use the the communicability matrix $\mathbf{G}$ with elements $G_{p q}$ between each pair of nodes $p$ and $q$. The communicability describes roughly how (e.g. information) content of node $p$ flows to node $q$ [19-21]. Here, we use slightly modified communicability $\mathbf{G}$ where the first M-1 walks are removed and define it as

$$
\mathbf{G}(\beta ; M)=\sum_{k=M}^{\infty} \frac{\beta^{k} \mathbf{A}^{k}}{k !}=\operatorname{Exp}[\beta \mathbf{A}]-\left(\mathbf{1}+\sum_{k=1}^{M-1} \frac{\beta^{k} \mathbf{A}^{k}}{k !}\right)
$$

We use here $M=5$ which excludes the simplest levels $L \leq 4$ from counting of walks. In practice, the analysis is not sensitive to choice of $M$ provided it remains below $M=5$, since in these lower levels the walks do not include the connection that are established and level of walks longer than 6 , through the cotexts and contexts. In the lower levels and with $M \leq 5$ the analysis essentially coincides with the word-frequency analysis. The parameter $\beta$ is used to tune how extensive apart of the network is included in counting the walks. By varying the parameter $\beta$ we get information how meaning attached to terms changes when only sentence-level connections are included ( $\beta$ low) in comparison to case when contexts level is taken into account ( $\beta$ high). It should be noted that due to finite size of the networks, after certain high enough value of $\beta$ the contribution from context-level does not change anymore. An optimum value of parameter $\beta$ is such that all paths that increase the diversity of key terms and words that contribute to the total communicability are included with the lowest possible value of $\beta$. This corresponds roughly to the maximum value of the Frechet-derivative [19] of $\mathbf{G}$ occurring with values $1.5<\beta<3.0$ in the cases studied here. In what follows, the communicability is normalized to a maximum value of one. By using the (normalized) communicability $[\mathbf{G}]_{p q}=G_{p q}$ between nodes $p$ and $q$ we can now obtain the total lexical support of node $q$ from all other nodes $p$, which are taken to be relevant in providing the lexical meaning of it.

The communicability of nodes within the lexical network is next used as the basis to form the lexical proximity network. In the lexical proximity network we retain only those connections between nodes $p$ and $q$ that exceed a certain threshold $G^{*}$ of communicability. The lexical proximity network thus contains the most important lexical connections.

\subsection{Construction of Lexicon Profile}

The terms in the lexical proximity network are the basis to construct the lexicon profiles for the terms $\mathrm{T}=$ [electron] or $\mathrm{T}=$ [photon]. The key terms that are connected to $\mathrm{T}$ in the proximity network are classified in $\mathrm{N}$ categories, which condense the information of the lexical connections. Each category of the key 
terms describes a given descriptive property $\mathrm{P}=1,2 \ldots, \mathrm{N}$ of interest. This classification is made for the practical purpose of condensing the relevant lexical information, because lexical networks with complete interrelationships between terms are too rich to easily yield the relevant information of the lexical structure. The condensed representation of the lexicon in the form of a descriptive property of the words in a given category $\mathrm{P}$ and with information on the relative importance of that category is referred to as a lexicon profile in what follows.

The lexicon profile is formed by defining the lexical support $\Pi(P)$ the term $\mathrm{T}$ receives from the lexical proximity network of key terms $\mathrm{p}(\mathrm{P})$ attached to a given feature $\mathrm{P}$. Such support is operationalized as the sum of closeness centrality $C C_{p}(P ; T)$ of node $p(\mathrm{P})$ in proximity network of term $\mathrm{T}$, in the form

$$
\Pi_{P}(T)=\sum_{p \in P} C C_{p}(P ; T)
$$

In Eq. (2) the closeness centrality $C C_{p}$ of node $p$ is defined in the standard way [19]. The components $\Pi_{P}$ form the lexical profile as an $\mathrm{N}$-dimensional vector of lexical supports $\bar{\Pi}(T)=\left(\Pi_{1}, \Pi_{2}, \ldots, \Pi_{N}\right) / \operatorname{Max}\left[\left\{\Pi_{P}\right\}\right]$ where the normalization make the lexicon profiles of different texts comparable.

\section{Results}

The lexical proximity networks of texts A and B are shown in Figs. 1 and 2, respectively. In the lexical proximity networks, we have denoted (with symbols explained in Table 2) the nodes representing terms and words, which are relevant for lexicons of $\mathrm{T}=$ [electron] and $\mathrm{T}=$ [photon]. Note that the lexical proximity network are always specific to the term $\mathrm{T}$ of interest. The links in the network correspond to communicability between nodes that exceeds the threshold value, which is chosen to be approximately $G^{*}=0.1 \beta$, with $\beta \geq 1$ (note that with increasing $\beta$ the values of communicabilities increase). The closeness centrality of nodes in the proximity network is shown as the size of the node.

The parameter $\beta$ controls the extent of walks included in the analysis. The highest value $\beta \approx 2.5$ corresponds to the maximum of the Frechet-derivative of the total communicability, and with increasing $\beta$ no essentially new connections are available. However, up to this value, with increased values of $\beta$, the closeness of the nodes change, revealing that more remote connections start to contribute to the communicability. In the case of the stratified network construction (as explained in Table 1) this means that for $\beta=1$ mainly the lexical at level $\mathrm{L}$ from 4 up to 5 is included, while for $\beta=2.5$ levels $\mathrm{L} \geq 7$ also contribute to the communicability of nodes. These levels $\mathrm{L} \geq 7$ bring in the semantic, contextrelated connections.

The lexicons for speaking about electrons and photons are here taken to consist of terms connected to nine $(\mathrm{N}=9)$ different types of properties which are relevant in the contexts of the terms electron and photon. Examples of key words related to these properties are listed in Table 2. The nine properties $\mathrm{P}$ of interest here are: 
1. (W) Field and/or wave-properties and interference

2. (P) Particle properties, particle models, particle-like existence

3. (D) Dualistic nature of entities and wave-particle dualism

4. (e) Epiphenomenal nature of entities (i.e. exists only in localization/ measurement)

5. (Q) Quanta and quantization, quantum nature of excitations

6. (m) Classical mechanics related properties (e.g energy and linear momentum)

7. (S) Stochastic and probabilistic properties and indeterminacy

8. (N) Nomic (law-like) properties that refer to theory, laws and principles

9. (X) Experiment-related properties (in double-slit experiments)

The results in Fig. 1 show that the lexical proximity networks of text A attached to $\mathrm{T}=$ [electron] and $\mathrm{T}=$ [photon] have many similarities and their lexical networks overlap because many nodes play similar roles in them. However there are also several terms attached to [electron] and to [photon] which are not shared but which exceed the threshold $G^{*}$ for communicability. Such terms and words are specific to given $\mathrm{T}$ and thus important, although their closeness to $\mathrm{T}$ in the proximity network is not very high. In general, text $\mathrm{A}$ uses rather symmetric vocabulary for [electron] and [photon]. Some of the properties like Q and $\mathrm{X}$ appear to be very dominant in their lexicon profiles.

The results in Fig. 2 for text B show that in B the lexical networks for [electron] and [photon] are somewhat more limited than in A. In addition, the differences between lexical proximity networks for [electron] and [photon] appear to be larger in B than in A. The vocabulary in B does not show dominance of properties $\mathrm{Q}$ and $\mathrm{D}$, instead, property $\mathrm{S}$ is strongly present.

On the basis of the lexical proximity network we form the lexicon profiles that the texts $\mathrm{A}$ and $\mathrm{B}$ attach to [electron] and [photon] and reduce them to nine-dimensional vectors, where each dimension is denoted by one of the tags $\mathrm{P} \in\{\mathrm{F}, \mathrm{P}, \mathrm{D}, \mathrm{e}, \mathrm{Q}, \mathrm{m}, \mathrm{S}, \mathrm{N}, \mathrm{X}\}$ as they are indicated by the set $\mathrm{p}$ of key-words (for some examples see Table 2). The choice of key words is specific to the text, some of them identical, but generally different vocabularies are used. Therefore, the choice of key words contain an element of interpretation about the significance of the word.

The Fig. 3 shows the lexicon profiles corresponding to the lexical proximity networks in Figs. 1 and 2. The differences between the lexicon profiles corresponding to texts $\mathrm{A}$ and $\mathrm{B}$ are now clearly visible. Lexicon profiles for [electron] and [photon] in A are dominated by terms related to quanta and quantization (Q), and also words and terms related to dualism (D) and stochasticity (S) are in center of the vocabulary. The lexicon profiles are also very symmetric, revealing that [electron] and [photon] are addressed using very similar vocabulary. These features of the lexicon profiles are in line with the goal of text A, which is to discuss electrons and photons from a unified perspective and with emphasis on field quantization. 


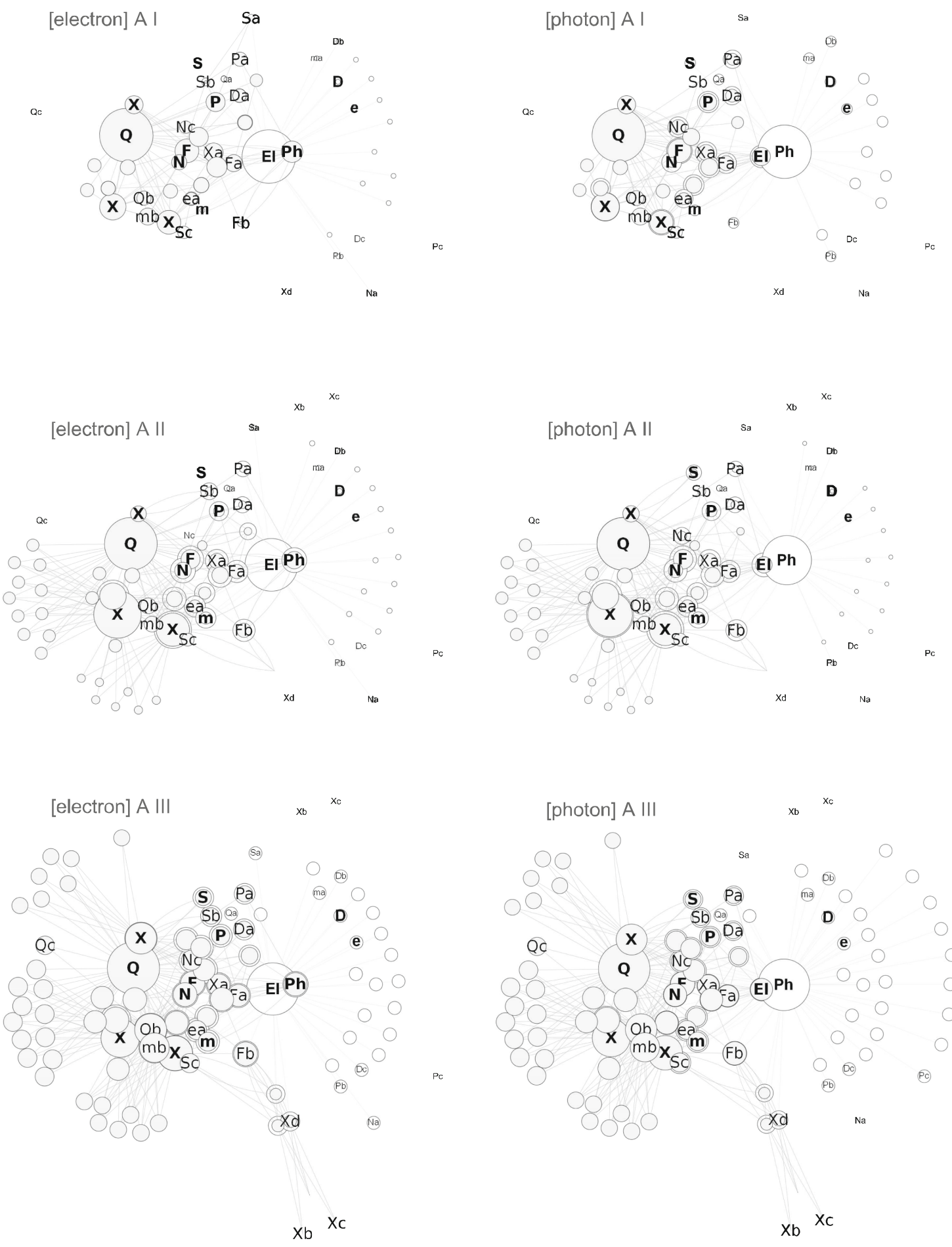

Fig. 1. The lexical proximity network for terms $T=$ [electron] and $T=$ [photon] as it appears in text A. The nodes corresponding to [electron] and [photon] are denoted by $\mathrm{El}$ and $\mathrm{Ph}$, respectively. In the left column, lexical proximity network for [electron] is shown in light gray and for the [photon] with white nodes. In the right column, [electron] is shown with white and [photon] with light gray nodes. The sizes of the nodes correspond to the closeness centrality of the node. The results are shown for $\beta$ $=1.0$ (I), 2.0 (II) and 2.5 (III). The threshold value for the communicability between the nodes, which is the basis of forming the proximity network, is $G^{*}=0.1 \beta$. 

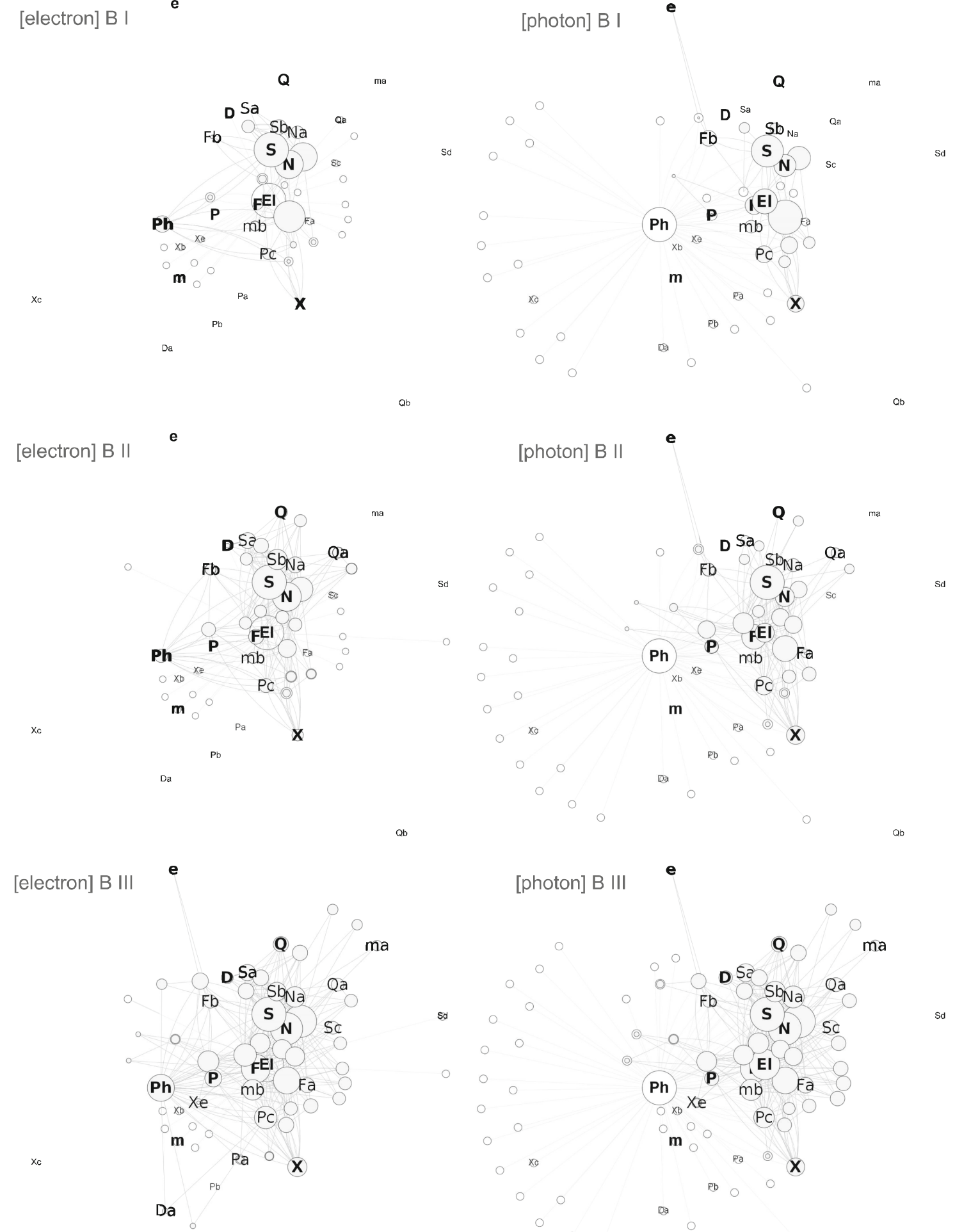

Fig. 2. The lexical proximity network for terms $\mathrm{T}=$ [electron $]$ and $\mathrm{T}=[$ photon $]$ as it appears in text B. The nodes corresponding to [electron] and [photon] are denoted by $\mathrm{El}$ and $\mathrm{Ph}$, respectively. In the left column, lexical proximity network for [electron] is shown in light gray and for [photon] with white nodes. In the right column, [electron] is shown with white and [photon] with light gray nodes. The sizes of the nodes correspond to the closeness centrality of the node. The results are shown for $\beta=1.0$ (I), 2.0 (II) and 2.5 (III). The threshold value for the communicability between the nodes, which is the basis of forming the proximity network, is $G^{*}=0.1 \beta$. 
Table 2. The $\mathrm{N}=9$ properties $\mathrm{P}$ defining the lexicon profiles and examples of the key words and terms attached to the properties. The most central key-word/term of the given property in texts $\mathrm{A}$ and $\mathrm{B}$ are denoted by subscripts, while the second and third most important additional key-words are denoted by subscript ADD. The symbols from $\mathrm{F}$ to $\mathrm{X}$ correspond to those in Figs. 1, 2 and 3.

\begin{tabular}{l|l|l|l|l}
\hline Property $\mathrm{P}$ & & \multicolumn{4}{|l}{ Key words and terms $\mathrm{p}$ for $\mathrm{P}$} \\
\cline { 4 - 5 } & & $\mathrm{p}_{\mathrm{A}}$ & $\mathrm{p}_{\mathrm{B}}$ & $\mathrm{p}$ ADD \\
\hline Field/Wave & $\mathrm{F}$ & field & wave & interference, interf/extend. pattern \\
\hline Particle & $\mathrm{P}$ & particle & particle & particle track, atom, object \\
\hline Dualism & $\mathrm{D}$ & field-partc. dual & wave-partc. dual & paradox, partc.-like \\
\hline Epiphenom & $\mathrm{e}$ & excitation & emergence & apparent particle, localization \\
\hline Quantization & $\mathrm{Q}$ & quantum & quantumobject & quantumstate, quantized field \\
\hline Mechanics & $\mathrm{m}$ & energy & monoenergetic & linear momentum, superposition \\
\hline Stochastics & $\mathrm{S}$ & probability & (prob. of) impacts & prob. density/distribution/amplit. \\
\hline Nomic & $\mathrm{N}$ & wavefunction & wavefunction & quantum mech./phys./theory. \\
\hline Experiments & $\mathrm{X}$ & detector/screen & interferometer & two-slit exp, electron/light beam \\
\hline
\end{tabular}

The lexicon profiles in $\mathrm{B}$, on the other hand, are clearly different from the profiles in A. Apart the fact that in B the stochastic (S) dimension of the lexicon profile is pronounced for both [electron] and [photon] as well, the lexicon profiles in B are quite different from those in $\mathrm{A}$. It is noteworthy that in B vocabulary for speaking about quanta and quantization is rather weak, as well as for dualism. In addition, the role of experiments for electrons and photons is asymmetric in $\mathrm{B}$; dominant for [photon] but weak for [electron]. These notions are in agreement with the general tone of text $\mathrm{B}$, which emphasizes the statistical interpretation of measurement events, a viewpoint which closely resembles the ensemble interpretation of quantum physics. The asymmetry of the role of experiments is clearly a consequence of B discussing interferometric experiments thoroughly for photons but not for electrons; for electrons no experimental results are discussed at all.

The lexicon profiles are shown in Fig. 3 for different values of $\beta$, corresponding to inclusion of different stratified levels of network. With increasing values from $\beta=1$ up to $\beta=2.5$ the analysis gradually covers levels from $\mathrm{L}$ from 4 and 5 up to levels $\mathrm{L} \leq 7$, where more remote connections start to contribute. These more remote connections increasingly provide the semantic, context related connections to the lexical terms. The more remote connections are supposedly important in providing the semantic content and also the different contextual ways to understand the meaning of terms; i.e. they reveal the context-relatedness and dependence of lexicons. 

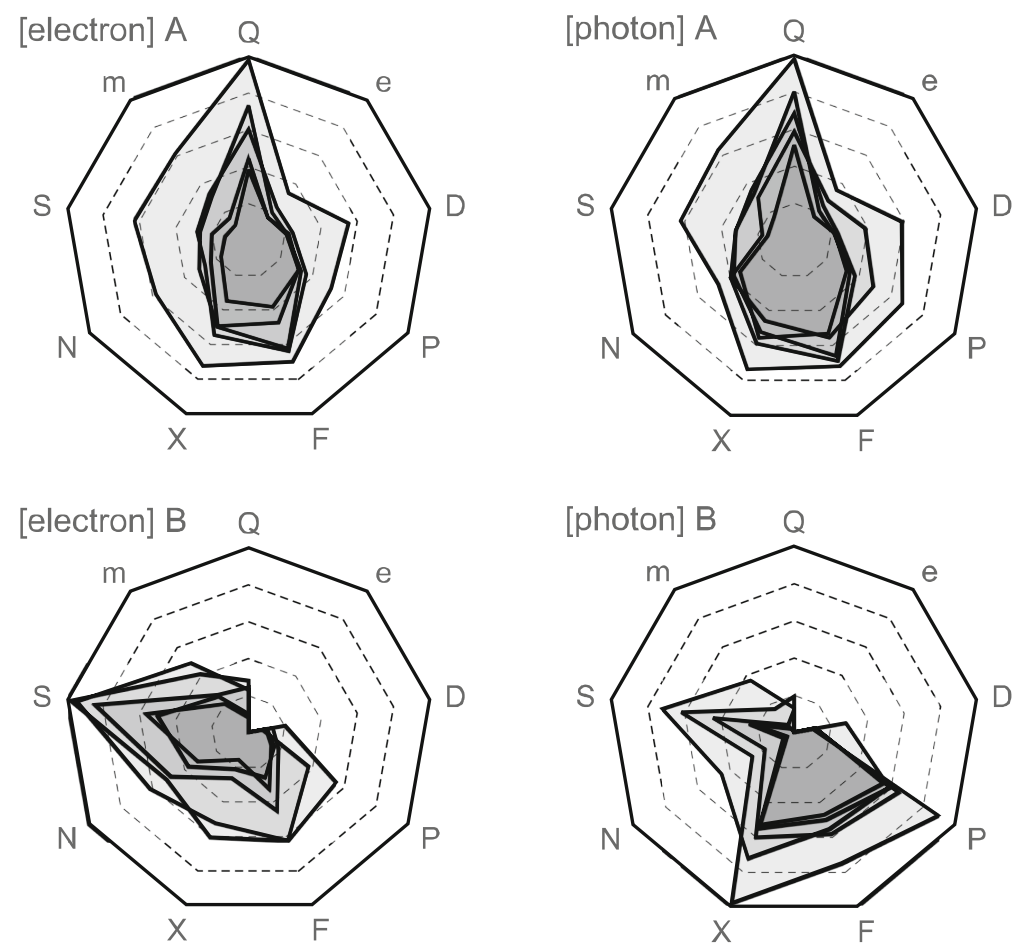

Fig. 3. The lexicon profiles for $\mathrm{T}=$ [electrons $]$ and $\mathrm{T}=$ [photons $]$ in texts $\mathrm{A}$ (the upper row) and $\mathrm{B}$ (the lower row). The symbols for the properties $\mathrm{P} \in\{\mathrm{F}, \mathrm{P}, \mathrm{D}, \mathrm{e}, \mathrm{Q}, \mathrm{m}, \mathrm{S}, \mathrm{N}, \mathrm{X}\}$ and how they are related to key words/terms is as in Table 2. Polygons show results for $\beta=$ $1.0,1.5,2.0$ and 2.5 , inner polygon corresponding to the lowest and the outmost to the highest value of $\beta$.

\section{Discussion and Conclusions}

The analysis of lexical networks and lexicon profiles is here used to reveal how didactical texts about the same topic may nevertheless use very different vocabularies. We used network-based methods to analyze two didactical texts about wave-particle dualism and nature of electrons and photons as quantum entities. Although the topic is the same, the texts analyzed here approach the topic differently. Therefore, they are suitable to test the method of analysis, which attempts to first construct the stratified lexical structure and then find the lexical proximity of terms in that structure.

The analysis presented here resembles more traditional analyses of semantic networks (compare with e.g. $[2,5,6]$ ). The difference, however, is that here we have performed stratified analysis, which is sensitive to features on the lexical level of cotext up to the level of contexts, where the lexical level meets the semantic level. In the lowest level, where connections are on sentence level, the results are expected to coincide with simple word-frequency counts. The results of the study show that the stratified analysis of lexical structure is able to reveal how the lexicons become augmented when the deeper contextual levels, by inclusion of more remote connections between the terms, are included in the analysis. The advantage of the method is thus the control it provides over the different level of connections. 
Regarding teaching and learning, an analysis of the lexical structures is an important starting point to better understand how lexicons affect what kinds of conceptions are conveyed in teaching and instruction, and how lexicons may either facilitate or hinder discussions of certain aspects of the topics to be learned. The didactical texts $\mathrm{A}$ and $\mathrm{B}$ discussed here clearly differ in how richly they cover vocabulary to discuss different aspects of the wave-particle duality. The text A has richer vocabulary, which is enriched when context level is taken into account so that the stochastic, dualistic and quantization dimensions S, D and Q are strengthened. This is as desired, since these dimensions are related to explanatory aspects, quite naturally a desirable feature of a didactical text. Still the symmetry of vocabulary needed to convey the symmetry in wave-particle dualism is maintained. The text $\mathrm{B}$, on the other hand, has more limited vocabulary, which also changes when context level is taken into account, but now mostly in dimensions particle $(\mathrm{P})$, field/wave $(\mathrm{F})$ and experiment $(\mathrm{X})$. These dimensions, however, are descriptive rather than explanatory. In addition, the vocabularies for electrons and photons are somewhat asymmetric. There is no indication in text B that such an asymmetric use of vocabulary was an intended features, given that the goal is wave-particle dualism. For a teacher who uses the didactical texts, either in teaching or for study, the ability to recognize and master rich enough terminology for many-faceted discussion is a very important component of professional competency. Therefore, it is also important to have tools to analyze didactical texts to become aware of such differences and to be able to detect them. To monitor the richness of lexicons used for didactical purposes, we need methods of research that are sensitive enough to features of different levels, from lexical to semantic levels, and which are controlled and reliable. For such purposes network-based methods provide new tools that complement and augment more traditional approaches.

Funding. This research was funded by the Academy of Finland, Grant 311449.

\section{References}

1. Darian, S.G.: Understanding the Language of Science. University of Texas Press, Austin (2003)

2. Yun, E., Park, Y.: Extraction of scientific semantic networks from science textbooks and comparison with science teachers' spoken language by text network analysis. Int. J. Sci. Educ. 40, 2118-2136 (2018)

3. Brookes, D.T., Etkina, E.: The importance of language in students' reasoning about heat in thermodynamic processes. Int. J. Sci. Educ. 37, 759-779 (2015)

4. Rincke, K.: It's rather like learning a language: development of talk and conceptual understanding in mechanics lessons. Int. J. Sci. Educ. 33, 229-258 (2011)

5. Clariana, R.B., Wolfe, M.M., Kim, K.: The influence of narrative and expository lesson text structures on knowledge structures: alternate measures of knowledge structure. Educ. Tech. Res. Dev. 62, 601-616 (2014)

6. Clariana, R.B., Wallace, P.E., Godshalk, V.M.: Deriving and measuring group knowledge structure from essays: the effects of anaphoric reference. Educ. Tech. Res. Dev. 57, 725-737 (2009) 
7. Derman, A., Eilks, I.: Using a word association test for the assessment of high school students' cognitive structures on dissolution. Chem. Educ. Res. Pract. 17, 902-913 (2016)

8. Neiles, K.Y., Todd, I., Bunce, D.M.: Establishing the validity of using network analysis software for measuring students' mental storage of chemistry concepts. J. Chem. Educ. 93, 821-831 (2016)

9. Kuhn, T.S.: The Road Since Structure. University of Chicago Press, Chicago (2000)

10. Hoyningen-Huene, P.: Reconstructing Scientific Revolutions. University of Chicago Press, Chicago (1993)

11. Evans, V.: How Words Mean: Lexical Concepts, Cognitive Models, and Meaning Construction. Oxford University Press, Oxford (2009)

12. Langacker, R.W.: Grammar and Conceptualization. Mouton de Gruyter, Berlin (1999)

13. Koponen, M., Asikainen, M.A., Viholainen, A., Hirvonen, P.E.: Using network analysis methods to investigate how future teachers conceptualize the links between the domains of teacher knowledge. Teach. Teach. Educ. 79, 137-152 (2019)

14. Kubsch, M., Nordine, J., Neumann, K., Fortus, D., Krajcik, J.: Probing the relation between students' integrated knowledge and knowledge-in-use about energy using network analysis. Eur. J. Math. Sci. Tech. Educ. 15, 1728 (2019)

15. Cheong, Y.W., Song, J.: Different levels of the meaning of wave-particle dualism and a suspensive perspective on the interpretation of quantum theory. Sci. Educ. 23, 1011-1030 (2014)

16. Ayene, M., Krick, J., Damitie, B., Ingerman, A., Thacker, B.: A holistic picture of physics student conceptions of energy quantization, the photon concept, and light quanta interference. Int. J. Sci. Math. Educ. 17, 1049-1070 (2019)

17. Hobson, A.: There are no particles, there are only fields. Am. J. Phys. 81, 211-223 (2013)

18. Müller, R., Wiesner, H.: Teaching quantum mechanics on an introductory level. Am. J. Phys. 70, 200-209 (2002)

19. Estrada, E.: The Structure of Complex Networks. Oxford University Press, Oxford (2012)

20. Koponen, I.T., Nousiainen, M.: Concept networks in learning: finding key concepts in learners' representations of the interlinked structure of scientific knowledge. J. Complex Netw. 2, 187-202 (2014)

21. Koponen, I.T., Nousiainen, M.: Concept networks of students' knowledge of relationships between physics concepts: finding key concepts and their epistemic support. Appl. Netw. Sci. 3, 14 (2018) 